\title{
Multiple solutions for a coercive quasilinear elliptic equation via Morse theory
}

\author{
Lifang $\mathrm{Fu}^{1}$ and Mingzheng Sun ${ }^{2^{*}}$ (D)
}

"Correspondence: suncut@163.com ${ }^{2}$ College of Sciences, North China University of Technology, Beijing 100144, People's Republic of China Full list of author information is available at the end of the article

\begin{abstract}
We study the quasilinear elliptic problem which is resonant at zero. By using Morse theory, we obtain five nontrivial solutions for the equation with coercive nonlinearities.
\end{abstract}

MSC: 35J92; 35J35; 35B34

Keywords: Quasilinear elliptic equations; Resonant; Morse theory

\section{Introduction}

Let $\Omega$ be a bounded domain in $\mathbb{R}^{N}(N \geq 1)$ with smooth boundary $\partial \Omega$. We study the following quasilinear elliptic problem:

$$
\begin{cases}-\Delta_{p} u-\Delta u=f(x, u) & \text { in } \Omega, \\ u=0 & \text { on } \partial \Omega,\end{cases}
$$

where $2<p<\infty$ and $\Delta_{p}$ denotes the $p$-Laplacian operator defined by

$$
\Delta_{p} u=\operatorname{div}\left(|\nabla u|^{p-2} \nabla u\right)
$$

In what follows, we denote by

$$
0<\lambda_{1}<\lambda_{2} \leq \cdots \leq \lambda_{k} \leq \cdots
$$

the eigenvalues of $-\Delta$ in $W_{0}^{1,2}(\Omega)$, and we let $\mu_{1}>0$ be the first eigenvalue of $-\Delta_{p}$ in $W_{0}^{1, p}(\Omega)$ (see [9]). Moreover, we make the following assumptions:

$\left(f_{1}\right) f \in \mathcal{C}^{1}(\bar{\Omega} \times \mathbb{R}, \mathbb{R})$ with $f(x, 0)=0$, and satisfies the following condition:

$$
\left|f^{\prime}(x, t)\right| \leq c\left(1+|t|^{q-2}\right), \quad \forall t \in \mathbb{R}, x \in \Omega,
$$

for some constants $c>0$ and $q \in\left[2, p^{*}\right)$, where $p^{*}=N p /(N-p)$ if $p<N$ and $p^{*}=+\infty$ if $N \leq p$,

(c) The Author(s) 2021. This article is licensed under a Creative Commons Attribution 4.0 International License, which permits use, sharing, adaptation, distribution and reproduction in any medium or format, as long as you give appropriate credit to the original author(s) and the source, provide a link to the Creative Commons licence, and indicate if changes were made. The images or other third party material in this article are included in the article's Creative Commons licence, unless indicated otherwise in a credit line to the material. If material is not included in the article's Creative Commons licence and your intended use is not permitted by statutory regulation or exceeds the permitted use, you will need to obtain permission directly from the copyright holder. To view a copy of this licence, visit http://creativecommons.org/licenses/by/4.0/. 
$\left(f_{2}\right)$ there exist $M>0$ and $\lambda<\frac{\lambda_{1}}{2}$ such that

$$
F(x, t)-\frac{1}{p} \mu_{1}|t|^{p} \leq \lambda|t|^{2}, \quad \text { for }|t| \geq M, x \in \Omega
$$

where $F(x, t)=\int_{0}^{t} f(x, s) d s$,

$\left(f^{-}\right)$there exist $\alpha>0$ and $k \geq 3$ such that

$$
f^{\prime}(x, 0)=\lambda_{k}, F(x, t) \leq \frac{1}{2} \lambda_{k} t^{2}, \quad \text { for }|t| \leq \alpha, x \in \Omega,
$$

$\left(f^{+}\right)$there exist $\alpha>0, k \geq 3, C>0$ and $2<\theta<p$ such that

$$
f^{\prime}(x, 0)=\lambda_{k}, F(x, t) \geq \frac{1}{2} \lambda_{k} t^{2}+C|t|^{\theta}, \quad \text { for }|t| \leq \alpha, x \in \Omega .
$$

Under the conditions above, from [14, Theorem 1.2] we know that Eq. (1.1) has at least four nontrivial solutions. It is worth pointing out that, using similar conditions, the authors in [10, Theorem 3.2] not only obtain four nontrivial solutions, but also prove that two of them are sign changing. Moreover, when the nonlinearity $f$ is resonant at infinity and non-resonant at zero, using variational methods, together with truncation and comparison techniques and Morse theory, the paper [11] can get the existence of six nontrivial solutions (two of them are sign changing).

The aim of this paper is to obtain the existence of another solution. Specifically, our result reads as follows.

Theorem 1.1 If $\left(f_{1}\right),\left(f_{2}\right)$ and $\left(f^{-}\right)\left(\right.$or $\left.\left(f^{+}\right)\right)$hold, then Eq. (1.1) has at least five nontrivial solutions.

Remark 1.2 (1) In our proof, we first obtain a nontrivial solution near zero inspired by papers $[13,15]$. Then we use the estimation of critical groups to distinguish the new solution from the known solutions of $[10,14]$. The method of estimating critical groups comes from [5], which has studied the bifurcation problem of semilinear elliptic equations at zero, and obtained six nontrivial solutions of the equation with coercive nonlinearities.

(2) Checking the proof below, our result is also true when $p=2$. So as far as we know, our theorem is new even for the semilinear elliptic equation.

This paper is organized as follows. In Sect. 2, by Morse theory the existence of a new nontrivial solution and the estimation of its critical groups are given. In Sect. 3, we give the proof of Theorem 1.1. In the sequel, the letter $C$ will be used indiscriminately to denote a suitable positive constant whose value may change from line to line.

\section{A solution near zero}

For any $\lambda \in \mathbb{R}$, let $f(x, u)=\lambda_{k} u+g(x, u)$ and $G(x, u)=\int_{0}^{u} g(x, s) d s$, then we consider the $\mathcal{C}^{2}$ functional $I_{\lambda}: W_{0}^{1, p}(\Omega) \rightarrow \mathbb{R}$ defined by setting

$$
I_{\lambda}(u)=\frac{1}{p} \int_{\Omega}|\nabla u|^{p} d x+\frac{1}{2} \int_{\Omega}|\nabla u|^{2} d x-\frac{\lambda}{2} \int_{\Omega}|u|^{2} d x-\int_{\Omega} G(x, u) d x,
$$


where $W_{0}^{1, p}(\Omega)$ is the Sobolev space endowed with the norm

$$
\|u\|=\|\nabla u\|_{p}=\left(\int_{\Omega}|\nabla u|^{p} d x\right)^{1 / p} .
$$

By $\left(f_{1}\right)$, weak solutions of Eq. (1.1) correspond to critical points of functional $I_{\lambda_{k}}$, which is also defined in the following form:

$$
I_{\lambda_{k}}(u)=\frac{1}{p} \int_{\Omega}|\nabla u|^{p} d x+\frac{1}{2} \int_{\Omega}|\nabla u|^{2} d x-\int_{\Omega} F(x, u) d x .
$$

By [6, Page 277], the second order differential of $I_{\lambda_{k}}$ in isolated critical point $u_{0}$ is given by

$$
\begin{aligned}
\left\langle I_{\lambda_{k}}^{\prime \prime}\left(u_{0}\right) v, w\right\rangle= & \int_{\Omega}\left(1+\left|\nabla u_{0}\right|^{p-2}\right)(\nabla v \nabla w) d x \\
& +\int_{\Omega}(p-2)\left|\nabla u_{0}\right|^{p-4}\left(\nabla u_{0} \nabla v\right)\left(\nabla u_{0} \nabla w\right) d x-\int_{\Omega} f^{\prime}\left(x, u_{0}\right) v w d x
\end{aligned}
$$

for any $v, w \in W_{0}^{1, p}(\Omega)$. In addition, if we assume that $I_{\lambda_{k}}\left(u_{0}\right)=c \in \mathbb{R}$, and $U$ is an isolated neighborhood of $u_{0}$, then the group

$$
C_{\ell}\left(I_{\lambda_{k}}, u_{0}\right)=H_{\ell}\left(I_{\lambda_{k}}^{c} \cap U, I_{\lambda_{k}}^{c} \cap U \backslash\left\{u_{0}\right\}\right), \quad \ell \in \mathbb{N}=\{0,1,2, \ldots\},
$$

is called the $\ell$ th critical group of the functional $I_{\lambda_{k}}$ at $u_{0}$, where $I_{\lambda_{k}}^{c}=\left\{u \in W_{0}^{1, p}(\Omega): I_{\lambda_{k}}(u) \leq\right.$ $c$ ), and $H_{\ell}(\cdot, \cdot)$ are the singular relative homological groups with a coefficient group $\mathbb{F}$ (see [1, Definition 4.1, Chapter I]).

Before stating our results, we recall the following result concerning critical groups estimates.

Lemma 2.1 ([8, Theorem 3.1]) Let $V$ be a subspace of $W_{0}^{1, p}(\Omega)$ of finite dimension $m$. For critical point $u_{0}$ of $I_{\lambda_{k}}$, we assume that:

(i) the function $I_{\lambda_{k}}$ is of class $C^{2}$ on $u_{0}+V$ and for every $v \in V$ the functions $u_{0} \mapsto\left\langle I_{\lambda_{k}}^{\prime}\left(u_{0}\right), v\right\rangle$ and $u_{0} \mapsto\left\langle I_{\lambda_{k}}^{\prime \prime}\left(u_{0}\right) v, v\right\rangle$ are continuous on $W_{0}^{1, p}(\Omega)$,

(ii) $\left\langle I_{\lambda_{k}}^{\prime \prime}\left(u_{0}\right) v, v\right\rangle<0$ for every $v \in V \backslash\{0\}$.

Then we have $C_{\ell}\left(I_{\lambda_{k}}, u_{0}\right)=\{0\}$ for every $\ell \leq m-1$.

Lemma 2.2 If $\left(f_{1}\right)$ and $\left(f^{-}\right)$hold, then Eq. (1.1) has a nontrivial solution $v_{0}$ such that

$$
C_{\ell}\left(I_{\lambda_{k}}, v_{0}\right)=\{0\}, \quad \forall \ell \leq d_{k-1}-1
$$

where $d_{k-1}=\operatorname{dim}\left\{\bigoplus_{i \leq k-1} \operatorname{ker}\left(-\Delta-\lambda_{i}\right)\right\}$.

Proof The proof will be divided into several steps.

(1) For some $\delta>0$ with $\lambda_{k}<\lambda<\lambda_{k}+\delta<\lambda_{k+1}$, we assume that

$$
K\left(I_{\lambda}\right)=\left\{u \in W_{0}^{1, p}(\Omega): I_{\lambda}^{\prime}(u)=0\right\}
$$


is the set of critical points of $I_{\lambda}$. By (2.1) and (2.2), we know that

$$
\left\langle I_{\lambda}^{\prime \prime}(0) v, w\right\rangle=\int_{\Omega} \nabla v \nabla w d x-\lambda \int_{\Omega} v w d x, \quad \forall v, w \in W_{0}^{1, p}(\Omega)
$$

then $I_{\lambda}^{\prime \prime}(0)$ is injective. So $u=0$ is an isolated critical point (see [7, Corollary 2.4]). Meanwhile, [6, Theorem 1.1] shows that

$$
C_{\ell}\left(I_{\lambda}, 0\right)=\delta_{\ell, d_{k}} \mathbb{F}
$$

For some small $\rho>0$, let

$$
B_{\rho}(0)=\left\{u \in W_{0}^{1, p}(\Omega):\|u\|<\rho\right\}
$$

be an isolated neighborhood of 0 such that $K\left(I_{\lambda}\right) \cap B_{\rho}(0)=\{0\}$. If we define $O=\overline{B_{\rho}(0)}$, then $I_{\lambda}$ satisfies the Palais-Smale condition on $O$. Let $\beta_{1}, \beta_{2}$ be regular values of $I_{\lambda}$ such that

$$
\beta_{1}<\inf _{O} I_{\lambda} \leq \sup _{O} I_{\lambda}<\beta_{2}
$$

Define

$$
W=O_{\beta_{1}}^{\beta_{2}}=\tilde{O} \cap I_{\lambda}^{-1}\left[\beta_{1}, \beta_{2}\right], \quad W^{-}=W \cap I_{\lambda}^{-1}\left(\beta_{1}\right),
$$

where $\tilde{O}=\bigcup_{t \in \mathbb{R}} \vartheta(t, O)$ and $\vartheta$ is the pseudo-gradient flow of $I_{\lambda}$. Here $\left(W, W^{-}\right)$is called the Gromoll-Meyer pair for the isolated critical point $u=0$ (see [1, Definition 5.1, Chapter I]). Meanwhile, using (2.4) and [1, Theorem 5.2, Chapter I] we have

$$
C_{\ell}\left(I_{\lambda}, 0\right)=H_{\ell}\left(W, W^{-}\right)=\delta_{\ell, d_{k}} \mathbb{F} .
$$

(2) For any $u, v \in W_{0}^{1, p}(\Omega)$ and $\lambda_{k}<\lambda<\lambda_{k}+\delta$ with $\delta>0$, using (2.1) we get

$$
\begin{aligned}
\left\|I_{\lambda}(u)-I_{\lambda_{k}}(u)\right\|_{\mathcal{C}^{1}(W)} & =\left\|I_{\lambda}-I_{\lambda_{k}}\right\|_{\mathcal{C}(W)}+\left\|I_{\lambda}^{\prime}-I_{\lambda_{k}}^{\prime}\right\|_{\mathcal{C}(W)} \\
& =\sup _{u \in W}\left|I_{\lambda}(u)-I_{\lambda_{k}}(u)\right|+\sup _{u \in W} \sup _{\|v\| \leq 1}\left|\left\langle I_{\lambda}^{\prime}(u)-I_{\lambda_{k}}^{\prime}(u), v\right\rangle\right| \\
& \leq \delta \sup _{u \in W} \int_{\Omega} u^{2} d x+\delta \sup _{u \in W} \sup _{\|v\| \leq 1}\left|\int_{\Omega} u v d x\right| \\
& \leq C \delta .
\end{aligned}
$$

Thus, for any $\varepsilon>0$, by (2.7) there is $\delta>0$ such that

$$
\left\|I_{\lambda}-I_{\lambda_{k}}\right\|_{\mathcal{C}^{1}(W)}<\varepsilon, \quad \text { for } \lambda \in\left(\lambda_{k}, \lambda_{k}+\delta\right)
$$

Using [3, Theorem III.4], we know that Gromoll-Meyer pairs are stable under small perturbation, then (2.8) implies that there exists $\delta>0$ such that $\left(W, W^{-}\right)$is still a GromollMeyer pair for $I_{\lambda_{k}}$ with the critical set

$$
S_{\lambda_{k}}=B_{\rho}(0) \cap K\left(I_{\lambda_{k}}\right)=W \cap K\left(I_{\lambda_{k}}\right) .
$$


Then (2.6) implies that

$$
C_{\ell}\left(I_{\lambda_{k}}, S_{\lambda_{k}}\right)=H_{\ell}\left(W, W^{-}\right)=\delta_{\ell, d_{k}} \mathbb{F}
$$

Here more properties of $C_{q}\left(I_{\lambda_{k}}, S_{\lambda_{k}}\right)$ can be found in [3, Definition II.1, Theorem III.3].

(3) Assume that $S_{\lambda_{k}}=\left\{v_{1}, v_{2}, \ldots, v_{n}\right\}$ for some $n \in \mathbb{N},\left(W_{j}, W_{j}^{-}\right)$is the Gromoll-Meyer pair for $v_{j}(j=1, \ldots, n)$, and $Q(t)$ is a formal series with nonnegative integral coefficients. By ([2, Page 414]) we have the Morse relation (or see [13, Proposition 2.7]):

$$
\sum_{j=1}^{n} \sum_{\ell} \operatorname{rank} H_{\ell}\left(W_{j}, W_{j}^{-}\right) t^{\ell}=\sum_{\ell} \operatorname{rank} H_{\ell}\left(W, W^{-}\right) t^{\ell}+(1+t) Q(t)
$$

this together with (2.9) implies that $I_{\lambda_{k}}$ has a critical point $u_{0}(\rho) \in W$ such that

$$
C_{d_{k}}\left(I_{\lambda_{k}}, u_{0}(\rho)\right) \neq 0
$$

Without losing generality, we assume that $u=0$ is an isolated solution of Eq. (1.1). Using $\left(f_{1}\right)$ and $\left(f^{-}\right)$, by [14, Theorem 1.1] or [12, Proposition 2.3] for $p=2$ we have

$$
C_{\ell}\left(I_{\lambda_{k}}, 0\right)=\delta_{\ell, d_{k-1}} \mathbb{F}
$$

Then (2.11) and (2.12) show that Eq. (1.1) has a nontrivial solution $u_{0}(\rho) \in W$.

(4) By standard elliptic regularity arguments we have $u_{0}(\rho) \in \mathcal{C}_{0}^{1}(\bar{\Omega})$ (see [6, Page 277]). Since

$$
\left\|u_{0}(\rho)\right\|_{\mathcal{C}_{0}^{1}(\bar{\Omega})} \rightarrow 0, \quad \text { as } \rho \rightarrow 0,
$$

for any $\varepsilon>0$ there is some $\rho>0$ small enough such that

$$
\left\|u_{0}(\rho)\right\|_{\mathcal{C}_{0}^{1}(\bar{\Omega})}<\varepsilon
$$

Now, we choose constants $\lambda_{*}$ and $\lambda^{*}$ satisfying

$$
\lambda_{k-1}<\lambda_{*}<\lambda_{k}<\lambda^{*}<\lambda_{k+1}
$$

Let $\varepsilon>0$ to be selected suitably later. By $f^{\prime}(x, 0)=\lambda_{k}$, the compactness of $\bar{\Omega}$ and (2.13), we may find a solution $u_{0}(\rho)$ to our equation such that $\left\|u_{0}(\rho)\right\|_{\mathcal{C}_{0}^{1}(\bar{\Omega})}<\varepsilon$ and

$$
\lambda_{k-1}<\lambda_{*} \leq f^{\prime}\left(x, u_{0}(\rho)\right) \leq \lambda^{*}<\lambda_{k+1}, \quad \text { for all } x \in \bar{\Omega} \text {. }
$$


For $v \in E_{k-1} \backslash\{0\}$ where $E_{k-1}=\bigoplus_{i \leq k-1} \operatorname{ker}\left(-\Delta-\lambda_{i}\right)$, by (2.2), (2.13) and (2.14) we have

$$
\begin{aligned}
\left\langle I_{\lambda_{k}}^{\prime \prime}(\right. & \left.\left.u_{0}(\rho)\right) v, v\right\rangle \\
= & \int_{\Omega}\left(1+\left|\nabla u_{0}(\rho)\right|^{p-2}\right)|\nabla v|^{2} d x+\int_{\Omega}(p-2)\left|\nabla u_{0}(\rho)\right|^{p-4}\left(\nabla u_{0}(\rho) \nabla v\right)^{2} d x \\
& -\int_{\Omega} f^{\prime}\left(x, u_{0}(\rho)\right) v^{2} d x \\
\leq & \int_{\Omega}(1+C \varepsilon)|\nabla v|^{2} d x+C \varepsilon \int_{\Omega}\left(\nabla u_{0}(\rho) \nabla v\right)^{2} d x-\int_{\Omega} f^{\prime}\left(x, u_{0}(\rho)\right) v^{2} d x \\
\leq & \int_{\Omega}(1+C \varepsilon)|\nabla v|^{2} d x+C \varepsilon \int_{\Omega}\left|\nabla u_{0}(\rho)\right|^{2}|\nabla v|^{2} d x-\int_{\Omega} f^{\prime}\left(x, u_{0}(\rho)\right) v^{2} d x \\
\leq & \int_{\Omega}(1+C \varepsilon)|\nabla v|^{2} d x-\int_{\Omega} f^{\prime}\left(x, u_{0}(\rho)\right) v^{2} d x \\
\leq & \frac{(1+C \varepsilon) \lambda_{k-1}-\lambda_{*}}{\lambda_{k-1}}|\nabla v|^{2} d x .
\end{aligned}
$$

Moreover, we can choose $\varepsilon>0$ such that

$$
(1+C \varepsilon) \lambda_{k-1}-\lambda_{*}<0
$$

this together with (2.15) shows that

$$
\left\langle I_{\lambda_{k}}^{\prime \prime}\left(u_{0}(\rho)\right) v, v\right\rangle<0, \quad \text { for } v \in E_{k-1} \backslash\{0\}
$$

then from Lemma 2.1 we get (2.3). Let $v_{0}=u_{0}(\rho)$, then we complete the proof.

Lemma 2.3 If $\left(f_{1}\right)$ and $\left(f^{+}\right)$hold, then Eq. (1.1) has a nontrivial solution $v_{0}$ such that (2.3) holds.

Proof For some $\delta>0$ small such that $\lambda_{k}-\delta<\lambda<\lambda_{k}$, by [6, Theorem 1.1] we get

$$
C_{\ell}\left(I_{\lambda}, 0\right)=\delta_{\ell, d_{k-1}} \mathbb{F}
$$

Using $\left(f_{1}\right)$ and $\left(f^{+}\right)$, from [14, Theorem 1.1], we have

$$
C_{\ell}\left(I_{\lambda_{k}}, 0\right)=\delta_{\ell, d_{k}} \mathbb{F}
$$

Similar to Lemma 2.2, Eq. (1.1) has a nontrivial solution $v_{0}$ such that (2.3) holds. We complete the proof.

Remark 2.4 (1) According to our proof, it is not difficult to find that any critical point in $W$ satisfies the critical group estimation (2.3).

(2) In the lemmas above, the method for the existence of a nontrivial solution is the same as that in $[13,15]$, but our result also has a new content: the estimation of the critical groups for this solution. 


\section{Proof of theorem}

Now we can give the proof of our theorem as follows.

Proof of Theorem 1.1 Under our assumptions, Ref. [14] has proved that $I_{\lambda_{k}}$ satisfies the Palais-Smale condition, and there are three nontrivial solutions $u_{i}(i=1,2,3)$. Moreover, two of them are local minima such that

$$
C_{\ell}\left(I_{\lambda_{k}}, u_{1}\right)=C_{\ell}\left(I_{\lambda_{k}}, u_{2}\right)=\delta_{\ell, 0} \mathbb{F},
$$

and $u_{3}$ is the mountain pass solution such that (see [11] or [10, Page 412])

$$
C_{\ell}\left(I_{\lambda_{k}}, u_{3}\right)=\delta_{\ell, 1} \mathbb{F}
$$

From Lemma 2.2 and Lemma 2.3, we know that

$$
C_{\ell}\left(I_{\lambda_{k}}, S_{\lambda_{k}}\right)=H_{\ell}\left(W, W^{-}\right)=\delta_{\ell, d} \mathbb{F}
$$

where $S_{\lambda_{k}}=B_{\rho}(0) \cap K\left(I_{\lambda_{k}}\right)=W \cap K\left(I_{\lambda_{k}}\right), d=d_{k}$ for $\left(f^{-}\right)$and $d=d_{k-1}$ for $\left(f^{+}\right)$.

Claim: $u_{i} \notin W$ for $i=1,2,3$.

Reasoning by contradiction, when $u_{i} \in W$, from Lemma 2.2, Lemma 2.3 and Remark 2.4, there is $\rho>0$ small enough such that

$$
C_{\ell}\left(I_{\lambda_{k}}, u_{i}\right)=\{0\}, \quad \forall \ell \leq d_{k-1}-1, i=1,2,3,
$$

which is in contradiction with (3.1) and (3.2) because of $k \geq 3$. Then the claim holds.

If $I_{\lambda_{k}}$ has only four nontrivial critical points: $v_{0}$ and $u_{i}$ for $i=1,2,3$, then, for $a<$ $\inf I_{\lambda_{k}}\left(K\left(I_{\lambda_{k}}\right)\right)$, [14, Lemma 4.1] gives the $\ell$ th critical group of $I_{\lambda_{k}}$ at infinity:

$$
C_{\ell}\left(I_{\lambda_{k}}, \infty\right)=H_{\ell}\left(W_{0}^{1, p}(\Omega), I_{\lambda_{k}}^{a}\right)=\delta_{\ell, 0} \mathbb{F}
$$

where $\left(W_{0}^{1, p}(\Omega), I_{\lambda_{k}}^{a}\right)$ is the Gromoll-Meyer pair for $K\left(I_{\lambda_{k}}\right)$ (see [4, Theorem 2.2]).

Now from (3.1) to (3.4), using the Gromoll-Meyer pairs $\left(W, W^{-}\right)$and $\left(W_{0}^{1, p}(\Omega), I_{\lambda_{k}}^{a}\right)$, the Morse relation (2.10) gives

$$
(-1)^{d}+(-1)^{0}+(-1)^{0}+(-1)^{1}=(-1)^{0}
$$

this contradiction implies that Eq. (1.1) has another nontrivial solution $u_{4} \notin W$. The proof is completed.

\section{Conclusions}

Using the critical groups estimates, our theorem can get more nontrivial solutions. The main results presented in this paper improve and generalize the results in $[10,14]$. 


\section{Funding}

The paper was supported by NCUT (1 10052971921/102, 110052972027/014, XN137/002/006), KZ202010028048, and NSFC $(11771302,11671026)$.

Availability of data and materials

Not applicable.

\section{Competing interests}

The authors declare that they have no competing interests.

\section{Authors' contributions}

All authors contributed equally and significantly in writing this article. All authors read and approved the final manuscript.

\section{Author details}

${ }^{1}$ Faculty of Science, Henan University of Animal Husbandry and Economy, Zhengzhou 450044, People's Republic of China.

${ }^{2}$ College of Sciences, North China University of Technology, Beijing 100144, People's Republic of China.

\section{Publisher's Note}

Springer Nature remains neutral with regard to jurisdictional claims in published maps and institutional affiliations.

Received: 17 August 2020 Accepted: 7 February 2021 Published online: 19 February 2021

\section{References}

1. Chang, K.-C.: Infinite Dimensional Morse Theory and Multiple Solution Problems. Birkhäuser, Boston (1993)

2. Chang, K.-C.: Methods in Nonlinear Analysis. Springer, Berlin (2005)

3. Chang, K.-C., Ghoussoub, N.: The Conley index and the critical groups via an extension of Gromoll-Meyer theory. Topol. Methods Nonlinear Anal. 7, 77-93 (1996)

4. Chang, K.-C., Wang, Z.-Q.: Notes on the bifurcation theorem. J. Fixed Point Theory Appl. 1, 195-208 (2007)

5. Chen, Y., Su, J., Sun, M., Tian, R.: Multiple solutions for the coercive semilinear elliptic equations. J. Math. Anal. Appl. 487, $124031(2020)$

6. Cingolani, S., Vannella, G.: Critical groups computations on a class of Sobolev Banach spaces via Morse index. Ann. Inst. Henri Poincaré, Anal. Non Linéaire 20, 271-292 (2003)

7. Cingolani, S., Vannella, G.: Marino-Prodi perturbation type results and Morse indices of minimax critical points for a class of functionals in Banach spaces. Ann. Mat. Pura Appl. 186, 155-183 (2007)

8. Lancelotti, S.: Morse index estimates for continuous functionals associated with quasilinear elliptic equations. Adv. Differ. Equ. 7, 99-128 (2002)

9. Lindqvist, P.: On the equation $\operatorname{div}\left(|\nabla u|^{p-2} \nabla u\right)+\lambda|u|^{p-2} u=0$. Proc. Am. Math. Soc. 109, 157-164 (1990)

10. Papageorgiou, N.S., Rădulescu, V.D.: Qualitative phenomena for some classes of quasilinear elliptic equations with multiple resonance. Appl. Math. Optim. 69, 393-430 (2014)

11. Papageorgiou, N.S., Smyrlis, G.: On nonlinear nonhomogeneous resonant Dirichlet equations. Pac. J. Math. 264, 421-453 (2013)

12. $\mathrm{Su}, \mathrm{J}$ : Semilinear elliptic boundary value problems with double resonance between two consecutive eigenvalues. Nonlinear Anal. 48, 881-895 (2002)

13. Sun, M., Su, J., Zhang, B.: Critical groups and multiple solutions for Kirchhoff type equations with critical exponents. Commun. Contemp. Math. 2050031 (2020). https://doi.org/10.1142/S0219199720500315

14. Sun, M., Zhang, M., Su, J.: Critical groups at zero and multiple solutions for a quasilinear elliptic equation. J. Math. Anal. Appl. 428, 696-712 (2015)

15. Tian, R., Sun, M., Zhao, L.: Applications of Morse theory to some nonlinear elliptic equations with resonance at zero. Nonlinear Anal. 113, 87-93 (2015)

\section{Submit your manuscript to a SpringerOpen ${ }^{\circ}$ journal and benefit from:}

- Convenient online submission

- Rigorous peer review

- Open access: articles freely available online

- High visibility within the field

- Retaining the copyright to your article

Submit your next manuscript at $\boldsymbol{~ s p r i n g e r o p e n . c o m ~}$ 